mally develop in embryo sac with defective antipodal complex. The antipodal complex is located between the endosperm and the maternal tissues of ovule. The main function of antipodal cells with polytene chromosomes is synthesis of substances for the formation and protection of endosperm syncytium. The antipodal cells of Poaceae pass through consecutive stages of proliferation, differentiation and death. During their differentiation, antipodals nourish the emerging endosperm and the endosperm cellularization induces antipodal cell death. Speculated, the functioning of polyploid antipodal cells gives Poaceae an evolutionary advantage over other plants, because Poaceae is a most widespread group of plants on the Earth. Moreover, antipodal complex is a unique model for understanding the structure of polytene chromosomes. The aim of our work was to study the morphology of the nuclei of antipodal cells isolated from fertilized ovules (containing an inducer of cell death, endosperm) and unfertilized ovules (without endosperm) at different stages of programmed cell death. The work was performed on total specimens of embryo sacs isolated from fixed wheat ovules. We used methods of light microscopy (DAPI staining, acridine orange staining, Ag-Nor staining, immunocytochemical staining by the antibodies to fibrillarin, TUNEL assay) and transmission electron microscopy. We demonstrated that in fertilized embryo sacs during the differentiation, individual giant polytene chromosomes are formed in the nuclei of antipodal cells. During the death, chromosomes become more condensed and come together. Components of nucleoli segregate into the cavities of chromosomes, chromosomes become indistinguishable. Some nuclei can be strongly flattened and oblong. Dense chromatin of some nuclei may be fragmented. Then antipodal cells strongly converge and are absorbed by the endosperm. In the nuclei of antipodal cells from unfertilized embryo sacs, individual chromosomes can be detected at the stage of differentiation. During the death, chromosomes condense, in some cells, chromosomes come together, but complete integration of all chromosomes does not occur. In some nuclei, fragmentation of chromatin takes place, in others degradation of chromatin is observed in the central region of the nucleus. Segregation of the nucleolar components does not occur; nucleoli remain intact longer than the chromatin. Nuclei of antipodals from fertilized and unfertilized embryo sacs at the stage of the death are TUNEL-positive. To sum up, the morphology of the antipodal nuclei from fertilized and unfertilized ovules differs and has its own features.

doi: http://dx.doi.org/10.7124/bc.0009DB

\section{D-3. Transcription of pericentromeric tandemly repeated DNA transcripts in human preovulatory oocytes}
M. A. Dobrynin ${ }^{1,3}$, A. S. Kalugina ${ }^{3}$, N. M. Korchagina ${ }^{1}$, A. Prjibelski ${ }^{2}$, O. I. Podgornaya ${ }^{1,2}$, N. I. Enukashvily ${ }^{1 *}$.
${ }^{1}$ Institute of Cytology RAS, St. Petersburg, Rus- sia; ${ }^{2}$ St. Petersburg State University, Russia; ${ }^{3}$ ART Hospital AvaPeter-Scandinavia nie@newmail.ru

Only about 5 percent of DNA is made up of protein-coding genes; the other 95 percent is noncoding and referred as junk DNA. Most of 
the non-coding DNA is repeats either scattered through genome (dispersed repeats) or tandemly organized (tandem repeats, TR or satellite DNA). These repeats are transcribed and the transcripts are involved in different processes including early preimplantation embryogenesis. However it is still unknown are the tandem repeats transcripts accumulated during oogenesis or the repeats are transcribed de novo in early embryo prior after the genome activation. The aim of the study was to check the presence of pericentromeric TR transcripts in preovulatory oocytes and to reveal its intracellular distribution. Methods. The TR of pericentromeric satellite 3 (HS3) as well as its transcripts distribution in GV, MI and MII oocytes was studied with DNA-DNA and RNA-DNA FISH, respectively. The colocalisation of HS3 DNA and RNA with RNA-helicase p68 (DDX5) involved in the HS3 transcription regulation was estimated by immunoFISH. The GV and MI preovulatory oocytes were obtained from donors after the informed consent had been signed. GV and MI donor oocytes are not used for gametes banking and are usually discarded. They were donated for scientific purposes after the ethic committee permission was granted. The presence of HS3 transcripts in transcriptome was verified with bioinformatics' methods of analysis of published preovulatory oocytes transcriptome (Zhang et al., 2018). Results. The probe used was hybridized to pericentromeric regions of all chromosomes excluding 2,6,8,11,12,18,19 on lymphocytes chromosome spreads in DNA-DNA FISH experiments. In GV oocytes, the probe was revealed as the part of condensed heterochromatic ring included in the inverted karyosphere . In M1 and M2, the probe was localized to pericentromeric chromosome regions. In DNARNA FISH, the probe revealed $1 \mathrm{mkm}$ granules in ooplasm in M1, M2 but not in GV oocytes. Pretreatment with RNAse eliminated the hybridization signal. The HS3 RNA granules were associated with the granules of RNA helicase p68 (DDX5). The analysis of transcriptome revealed a polyadenilated HS3 transcript, that contained a sequence homological to the probe used in the experiment. The transcript contained also the sequence we describe previously as actively transcribed in senescent and malignant cells (Enukashvily et al., 2007). Conclusion. HS3 transcripts are accumulated in human oocyte after the transition from GV to M1. The transcripts are not associated with chromosomes but are adjacent to granules of RNA helicase p68.

The work was supported by grants from RFBR (18-3400279) and RSF (19-74-20102).

doi: http://dx.doi.org/10.7124/bc.0009DD

\section{F-1. AEDL peptide and $\mathrm{NaCl}$ affect on the ultrastructure and expression of DNA and lysine methyltransferase genes in Nicotiana tabacum L. regenerants}

\author{
L. I. Fedoreyeva ${ }^{1,2}$, E. N. Baranova ${ }^{2}$, \\ B. F. Vanyushin ${ }^{1,2}$ \\ ${ }^{1}$ AN. Belozersky Institute of Physico-Chemical \\ Biology, Lomonosov Moscow State University, \\ 119991 Moscow, Leninskie Gory1, building 40; \\ ${ }^{2}$ Institute of Agricultural Biotechnology RAS, \\ 127550 Moscow, Timiryazevskaya 42 \\ fedlara@inbox.ru
}

Due to its fixed nature, plants have a developed system of adaptation to environmental changes. The most studied epigenetic modifications of 